\title{
The Basic Principle of Phase-Transfer Catalysis and Some Mechanistic Aspects
}

Takuya Hashimoto and Keiji Maruoka

\section{1}

\section{Introduction}

In 1971, Starks introduced the term "phase-transfer catalysis" to explain the critical role of tetraalkylammonium or phosphonium salts $\left(\mathrm{Q}^{+} \mathrm{X}^{-}\right)$in the reactions between two substances located in different immiscible phases [1]. For instance, the displacement reaction of 1-chlorooctane with aqueous sodium cyanide is accelerated many thousand-fold by the addition of hexadecyltributylphosphonium bromide 1 as a phase-transfer catalyst (Scheme 1.1). The key element of this tremendous reactivity enhancement is the generation of quaternary phosphonium cyanide, which renders the cyanide anion organic soluble and sufficiently nucleophilic.

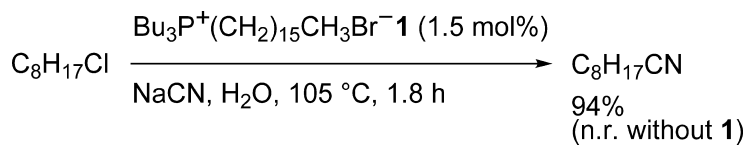

\section{Scheme 1.1}

Although this was not the first observation of the catalytic activity of quaternary onium salts, the foundations of phase-transfer catalysis were laid by Starks, together with Makosza and Brändström, during the mid to late 1960s [2]. Ever since that time, the chemical community has witnessed the steady growth of phase-transfer catalysis as a practical methodology for organic synthesis, featuring its simple experimental operations, mild reaction conditions, inexpensive and environmentally benign reagents and solvents, and the possibility to conduct large-scale preparations [3,4]. Nowadays, phase-transfer catalysis appears to be a prime synthetic tool, being appreciated not only in various fields of organic chemistry but also among widespread industrial applications.

Despite the development of phase-transfer catalysis in organic synthesis, the mechanistic aspects of phase-transfer catalysis remain obscure, due mainly to the 
difficulty of investigating biphasic systems and the many complex parameters that must be analyzed.

The aim of this chapter is to assist the reader to generate an intuitive understanding of the mechanism of asymmetric phase-transfer catalysis, together with a practical guide for the design of such processes. More detailed studies related to the physical and numerical aspects of phase-transfer catalysis may be consulted elsewhere [3].

\section{2}

\section{Inorganic Base-Promoted Activation of Acidic Organic Compounds}

The representative reaction system applied in asymmetric phase-transfer catalysis is the biphasic system composed of an organic phase containing an acidic methylene or methine compound and an electrophile, and an aqueous or solid phase of inorganic base such as alkaline metal ( $\mathrm{Na}, \mathrm{K}, \mathrm{Cs}$ ) hydroxide or carbonate. The key reactive intermediate in this type of reaction is the onium carbanion species, mostly onium enolate or nitronate, which reacts with the electrophile in the organic phase to afford the product.

\subsection{1}

\section{Generation of Reactive Onium Carbanion Species}

The exact pathway for generating the reactive onium carbanion species remains the subject of controversy, typically among Starks extraction mechanism (Scheme 1.2) and the Makosza interfacial mechanism (Scheme 1.3).

In the Starks extraction mechanism, the phase-transfer catalyst moves back and forth across the organic and aqueous phases. The onium salt equilibrates with the inorganic base in the aqueous phase, and extracts hydroxide into the organic phase.

Starks Extraction Mechanism

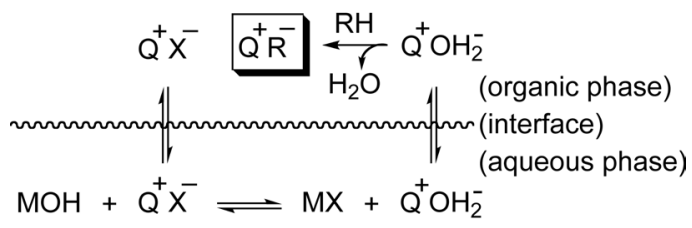

Scheme 1.2

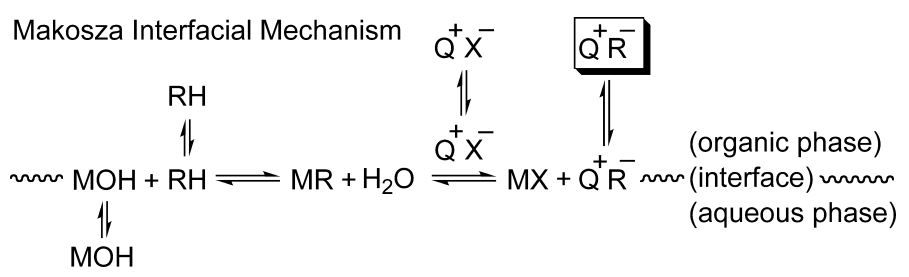

Scheme 1.3 
The onium hydroxide then abstracts hydrogen from the acidic organic compound to give the reactive intermediate $\mathrm{Q}^{+} \mathrm{R}^{-}$.

The advocated pathway of the interfacial mechanism is the first formation of metal carbanion at the interface of organic and aqueous phase in the absence of phasetransfer catalyst, followed by the extraction of the formed metal carbanion species from the interface into the organic phase by the action of phase-transfer catalyst.

Since asymmetric phase-transfer catalysts normally contain highly lipophilic chiral organic frameworks, and are reluctant to enter the aqueous phase, the Makosza interfacial mechanism seems plausible.

Clearly, the area of the interface and the basicity of the inorganic salt affect the amount of available onium carbanion. It should be also noted that an excessively lipophilic phase-transfer catalyst would hardly access the interface, and consequently the use of such a catalyst would result in an insufficient reaction.

\subsection{2}

\section{Stability of the Onium Carbanion}

The onium carbanion formed under phase-transfer conditions is unstable depending on the anion source, and in the absence of an electrophilic reaction partner, degradation of the accumulated onium carbanion in the organic phase may be observed. This is known to proceed via Hoffman elimination, nucleophilic substitution and/or Stevens rearrangement (Scheme 1.4) [4f,6,7]. The direct decomposition of onium salt, as influenced by the strong inorganic base at the interface, may be also operative.

\subsection{3}

\section{Reactivity of the Onium Carbanion}

Cation exchange from the metal cation to the onium carbanion improves the intrinsic reactivity of the latter due to formation of the "naked anion". At the same time, the

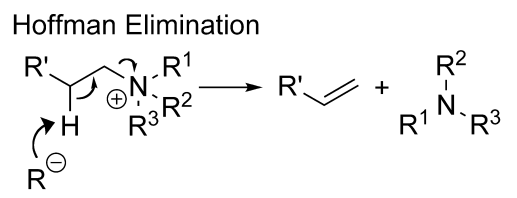

Nucleophilic Substitution

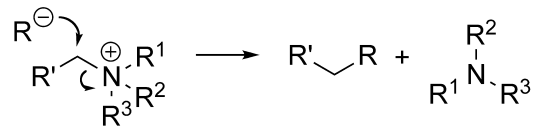

Stevens Rearrangement

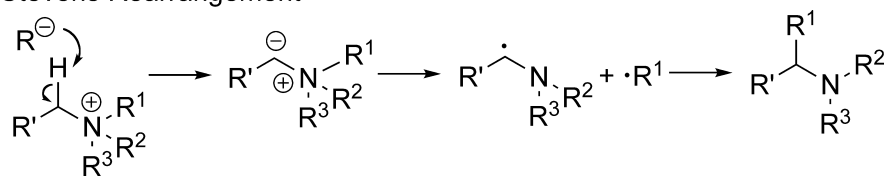

Scheme 1.4 
onium carbanion in the organic phase is less hydrated compared to the metal carbanion at the interface, which functions as another factor for an enhanced reactivity of the onium carbanion.

\section{2 .4}

\section{Nuclueophilic Substitution Reaction}

$$
Q^{+} R^{-}+R^{\prime} X \longrightarrow R^{\prime} R+Q^{+} X^{-}
$$

The fate of the onium carbanion $\mathrm{Q}^{+} \mathrm{R}^{-}$incorporated into the organic phase depends on the electrophilic reaction partner. The most studied area in the asymmetric phasetransfer catalysis is that of asymmetric alkylation of active methylene or methine compounds with alkyl halides, in an irreversible manner. The reaction mechanism illustrated above is exemplified by the asymmetric alkylation of glycine Schiff base (Scheme 1.5) [8].

In the first step, glycine Schiff base 2 reacts with the inorganic base at the interface of two phases to give the metal enolate 3 , which remains at the interface due to its highly polar character. The metal enolate 3 then exchanges the cation to provide onium enolate 4 . The sufficiently lipophilic 4 then moves into the organic phase to react with alkyl halide. After the reaction, onium halide is regenerated and enters the next catalytic cycle. The key issue to be considered here is the possibility of product racemization and dialkylation. In this example, the basicity of the inorganic base and acidity of the substrate and product, as well as other reaction conditions, are carefully adjusted to circumvent this problem. It should be also noted that control of the $E / Z$ geometry of the enolate is apparently critical to the asymmetric induction, although there is no clear evidence about which isomer is the actual reacting species in this case.

Asymmetric Alkylation of Glycine Schiff Base
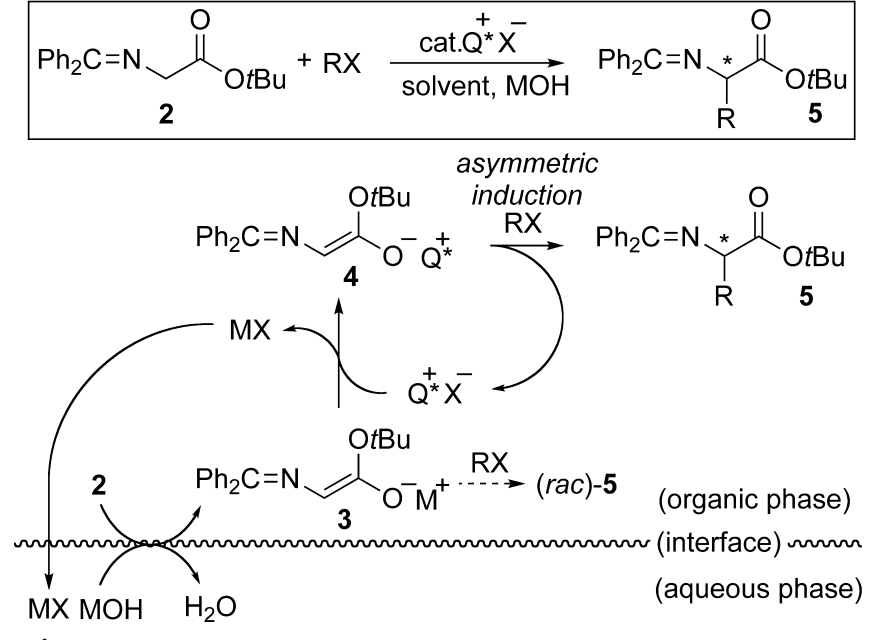

Scheme 1.5 
An asymmetric $\mathrm{S}_{\mathrm{N}} 2^{\prime}$ reaction and $\mathrm{S}_{\mathrm{N}} \mathrm{Ar}$ reaction are included as other examples of this category.

\section{2 .5}

\section{Nucleophilic Addition to Electrophilic $\mathrm{C}=\mathrm{X}$ Double Bonds}

$$
Q^{+} R^{-}+C_{C}=x \longrightarrow Q^{+} x^{-}-C-R
$$

Unlike the nucleophilic substitution reactions which generate stable onium halide after the reaction, nucleophilic additions to electrophilic $\mathrm{C}=\mathrm{X}$ double bonds $(\mathrm{X}=\mathrm{C}, \mathrm{N}, \mathrm{O})$ provide rather basic onium anion species as an initial product. If the anion is sufficiently stable under the reaction conditions, onium anion will then exchange the counter ion for the other metal carbanion at the interface to regenerate the reactive onium carbanion $\mathrm{Q}^{+} \mathrm{R}^{-}$. In another scenario, the basic onium anion may abstract the acidic hydrogen atom of the other substrate to provide $\mathrm{Q}^{+} \mathrm{R}^{-}$directly. Such a reaction system ideally requires only a catalytic amount of the base although, in general, a substoichiometric or excess amount of the base is used to lead the reaction to completion. An additional feature of this system is the substantial possibility of a retro-process at the crucial asymmetric induction step, which might be problematic in some cases.

The direct asymmetric aldol reaction under phase-transfer conditions is a representative example of this class of phase-transfer reaction, which is known to proceed with a catalytic amount of base and to include an undesired retro-process (Scheme 1.6) [9]. Here, the onium enolate 4 reacts with aldehyde in the organic

Direct Asymmetric Aldol Reaction of Glycine Schiff Base
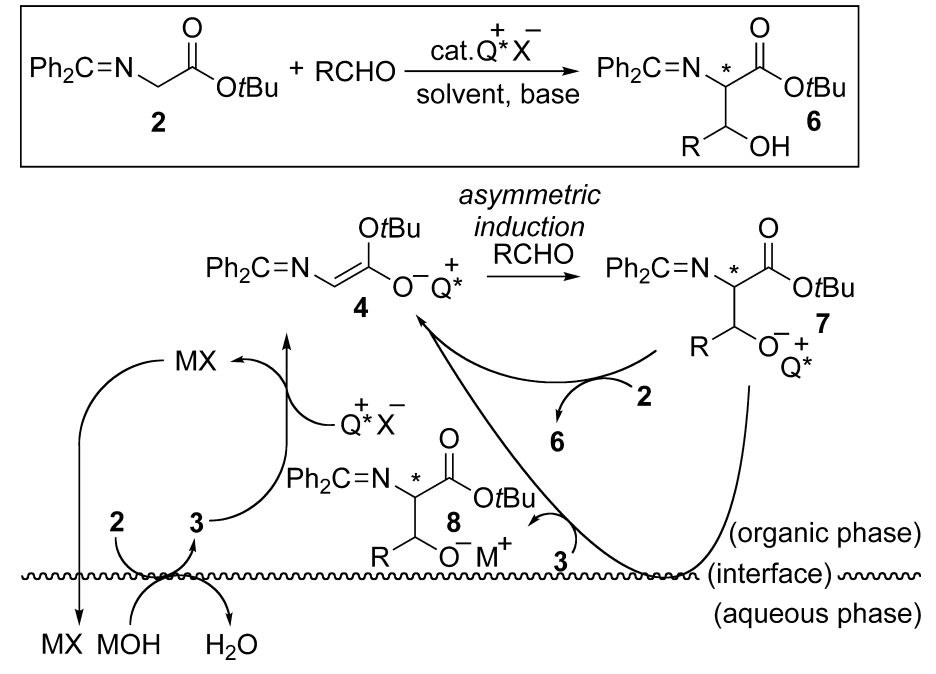

Scheme 1.6 
phase to give the onium alkoxide 7, which may abstract the hydrogen from the other glycine Schiff base 2 to provide another reactive intermediate onium enolate 4 and the protonated aldol product $\mathbf{6}$. It should be noted that this is not the only expected pathway, and there are complex equilibriums in this reaction system which, for reasons of clarity, not fully delineated below. The phase-transfer-catalyzed asymmetric Michael addition and the Mannich reaction are other typical examples which fall into this category.

\section{3}

\section{Phase-Transfer-Catalyzed Addition of Anion Supplied as Metal Salt}

The common feature in the asymmetric phase-transfer catalysis introduced above is formation of the reactive onium carbanion from the inorganic base and active methylene or methine compounds, followed by extraction of the carbanion species as the onium salt from the interfacial area into the organic phase. Thus, both reagents normally remain in the organic phase. In the other category of asymmetric phasetransfer catalysis, the anion itself supplied as an aqueous solution or solid of its inorganic salt as the reaction partner, and the two reactants are strictly separated by the interface. In such a system, the anion is transferred gradually from the aqueous phase into the organic phase by the intervention of an onium salt.

With regards to the mechanism of the generation of onium anion, the Starks extraction mechanism and interfacial mechanism (Brändström-Montanari modification) are suggested (Scheme 1.7). As in the above-described case, the interfacial mechanism seems to be operative in the asymmetric phase-transfer catalysis.

The reaction conditions are rather mild, so that the possibility of side reactions, such as catalyst decomposition, is considerably reduced. The major challenge associated with these reactions is an absence of prochirality in the anionic species. Namely, the chiral onium anion must discriminate the enantiotopic face of the distant electronically neutral reaction partner in the organic phase.

Asymmetric phase-transfer catalyzed oxidation of olefin using sodium hypochlorite or potassium permanganate as metal anion sources is the typical example of this category.

The asymmetric epoxidation of an $\alpha, \beta$-unsaturated ketone, using sodium hypochlorite, is illustrated in Scheme 1.8 [10]. Hypochlorite is extracted as an onium

Starks Extraction Mechanism

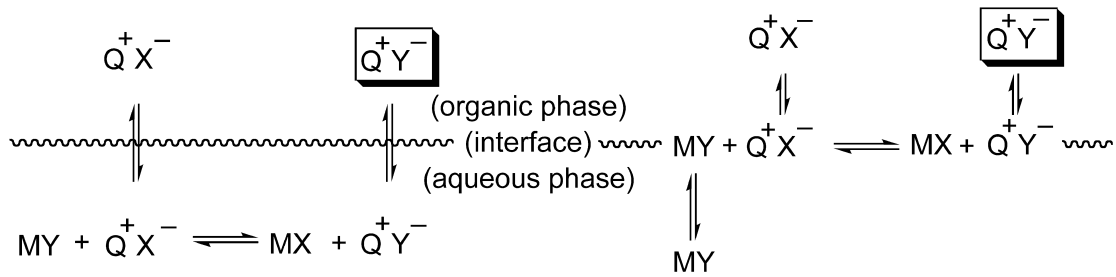

Scheme 1.7 
Asymmetric Epoxidation of $\alpha, \beta$-Unsaturated Ketones
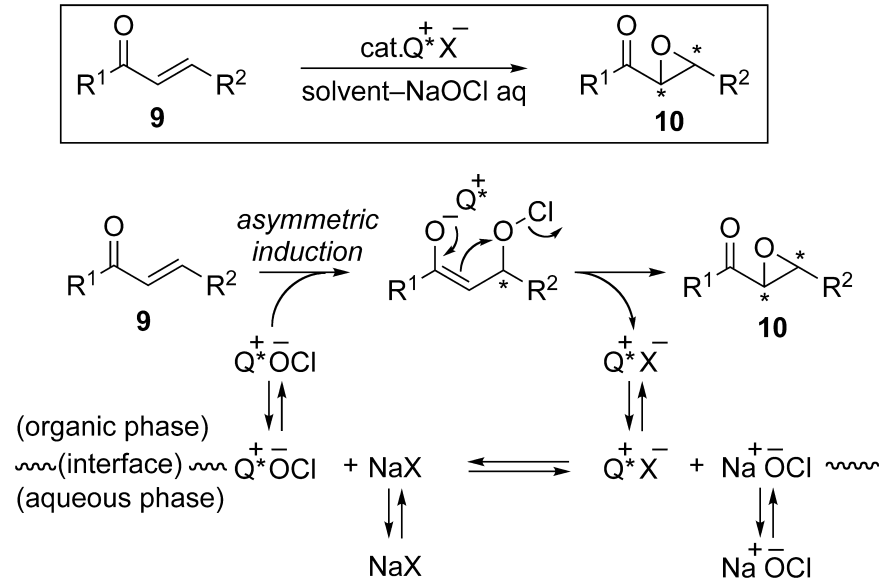

\section{Scheme 1.8}

salt into the organic phase, and the onium hypochlorite then reacts with the ketone while recognizing its enantiotopic face to provide the optically enriched $\alpha, \beta$-epoxy ketone.

\section{4}

\section{Use of Crown Ether as Phase-Transfer Catalyst}

Crown ether is the other important class of phase-transfer catalysts which critically differs from the onium salt, in that the whole inorganic salt is transferred into the organic phase. The reaction modes described above can generally be accommodated in such crown ether-catalyzed reactions, simply by replacing the onium cation by a metal cation complex of crown ether.

\section{References}

1 Starks, C.M. (1971) J. Am. Chem. Soc., 93, 195.

2 Makosza's pioneering contributions are representative, see: (a) Makosza, M. (1966) Tetrahedron Lett., 4621; (b) Makosza, M. (1966) Tetrahedron Lett., 5489; (c) Makosza, M. (1969) Tetrahedron Lett., 673;

(d) Makosza, M. (1969) Tetrahedron Lett., 677; see also: (e) Brändström, A. (1977) Adv. Phys. Org. Chem., 15, 267.
3 (a) Dehmlow, E.V. and Dehmlow, S.S. (1993) Phase Transfer Catalysis, 3rd edn. VCH, Weinheim; (b) Starks, C.M., Liotta, C.L. and Halpern, M.E. (1994) PhaseTransfer Catalysis, Chapman \& Hall, New York; (c) Sasson, Y. and Neumann, R. (eds), (1997) Handbook of Phase-Transfer Catalysis, Blackie Academic \& Professional, London; (d) Halpern, M.E. (ed.), (1997) Phase-Transfer Catalysis, ACS 
Symposium Series 659, American Chemical Society, Washington, DC.

4 For general reviews on asymmetric phasetransfer catalysis, see: (a) Shioiri, T. (1997) Handbook of Phase-Transfer Catalysis (eds Y. Sasson and R. Neumann), Blackie Academic \& Professional, London, Chapter 14; (b) O’Donnell, M.J. (1998) Phases - The Sachem Phase Transfer Catalysis Review, (4) p. 5; (c) O’Donnell, M.J. (1999) Phases - The Sachem Phase Transfer Catalysis Review, (5) p. 5; (d) Nelson, A. (1999) Angew. Chem., 111, 1685; Nelson, A. (1999) Angew. Chem. Int. Ed., 38, 1583; (e) Shioiri, T. and Arai, S. (2000) Stimulating Concepts in Chemistry (eds F. Vogtle, J.F. Stoddart and M. Shibasaki), Wiley-VCH, Weinheim, p. 123; (f) O’Donnell, M.J. (2000) Catalytic Asymmetric Syntheses (ed. I. Ojima), 2nd edn. Wiley-VCH, New York, Chapter 10; (g) O’Donnell, M.J. (2001) Aldrichimica Acta, 34, 3; (h) Maruoka, K. and Ooi, T. (2003) Chem. Rev., 103, 3013; (i) O’Donnell, M.J. (2004) Acc. Chem. Res., 37, 506; (j) Lygo, B. and Andrews, B.I. (2004) Acc. Chem. Res., 37, 518; (k) Vachon, J. and Lacour, J. (2006) Chimia, 60, 266; (1) Ooi, T. and Maruoka, K. (2007) Angew. Chem. Int. Ed., 46, 4222. 5 (a) Makosza, M. (1975) Pure Appl. Chem., 43, 439; (b) Yang, H.-M. and Wu, H.-S. (2003) Catal. Rev., 45, 463.

6 Patterson, D.E., Xie, S., Jones, L.A., Osterhout, M.H., Henry, C.G. and Roper, T.D. (2007) Org. Process Res. Dev., 11, 624.

7 Vial, L., Gonçalves, M.-H., Morgantini, P.-Y., Weber, J., Bernardinelli, G. and Lacour, J. (2004) Synlett, 1565.

8 Ooi, T., Kameda, M. and Maruoka, K. (2003) J. Am. Chem. Soc., 125, 5139, and references therein.

9 Ooi, T., Kameda, M., Taniguchi, M. and Maruoka, K. (2004) J. Am. Chem. Soc., 126, 9685, and references therein.

10 Ooi, T., Ohara, D., Tamura, M. and Maruoka, K. (2004) J. Am. Chem. Soc., 126, 6844 , and references therein. 